\title{
Skin Classification Scheme
}

National Cancer Institute

\section{Source}

National Cancer Institute. Skin Classification Scheme. NCI Thesaurus. Code C74562.

A data system used to specify attributes of the skin. 